\title{
La infancia y la adolescencia ante las Tecnologías de la Información y la Comunicación (TIC): oportunidades, riesgos y daño
}

\section{Haurrak eta nerabeak informazioaren eta komunikazioaren teknologien (IKT) aurrean: aukerak, arriskuak eta kalteak}

\section{Children and adolescents and Information and Communication Technologies (ICTs): opportunities, risks and harms}

\author{
Gemma Martínez ${ }^{\star}$, Maialen Garmendia, Carmelo Garitaonandia \\ Miembros del grupo de investigación EU Kids Online (UPV/EHU)
}

\begin{abstract}
RESUMEN: Los menores están creciendo en un sistema de convergencia mediática que les proporciona oportunidades para la socialización, la auto-expresión, el aprendizaje, la creatividad y la participación a través de los medios online y, crecientemente, los medios móviles. La experiencia digital de la infancia y la adolescencia se ha acentuado con el uso de smartphones y tabletas, así como con las smart TVs, videoconsolas, con los smartwatch, las aplicaciones que monitorizan la actividad fisica y otros recursos digitales que forman parte del Internet de las Cosas. Sin embargo, además de las oportunidades que ofrece Internet, los niños y niñas también experimentan riesgos evidenciando la interdependencia de ambos: cuanto más usan los menores internet, mayor es la gama de oportunidades que tienen y mayor es la probabilidad de exposición a experiencias de riesgo. Las oportunidades (comunicación, aprendizaje, creatividad, entretenimiento, etc.) y los riesgos online (ciberacoso, discurso del odio, pornografia, grooming, explotación comercial, etc.) van de la mano.
\end{abstract}

PALABRAS CLAVE: adolescencia, infancia, smartphones, Tecnologías de la Información, oportunidades, riesgos.

ABSTRACT: Children are growing up in a media convergence context that provides them with opportunities for socialization, self-expression, learning, creativity and participation through online media and, increasingly, mobile media. The digital experience of children and adolescents has been accentuated with the use of smartphones and tablets, as well as with smart TVs, game consoles, smartwatches, applications that monitor physical activity and other digital resources that are part of the Internet of things. However, the internet provides boys and girls with opportunities and risks and empirical evidence shows the interdependence of both: the more children use the Internet, the greater the range of opportunities they have and the greater the probability of exposure to risky experiences. Therefore, opportunities (communication, learning, creativity, entertainment, etc.) and online risks (cyberbullying, hate speech, pornography, grooming, commercial exploitation, etc.) go hand in hand.

KEYWORDS: adolescents, childrens, smartphones, information and communication technologies (ICTs), oportunities, risks, harms.

\footnotetext{
* Correspondencia a / Corresponding author: Gemma Martínez. Facultad de CC. Sociales de la UPV/EHU, Barrio Sarriena, s/n (48940 Leioa) - gemma.martinez@ehu.eus - https://orcid.org/0000-0001-6263-4238

Cómo citar / How to cite: Martínez, Gemma; Garmendia, Maialen; Garitaonandia, Carmelo (2020). «La infancia y la adolescencia ante las Tecnologías de la Información y la Comunicación (TIC): oportunidades, riesgos y daño", Zer, 25(48), 349-362. (https://doi.org/10.1387/zer.21116).

Recibido: 1 octubre, 2019; aceptado: 8 octubre, 2019.

ISSN 1137-1102 - eISSN 1989-631X / (c) 2020 UPV/EHU

(c)
} 


\section{Introducción}

Los menores están creciendo en un sistema de convergencia mediática (Ito et al., 2009) que les proporciona oportunidades para la socialización, la auto-expresión, el aprendizaje, la creatividad y la participación a través de los medios online y, crecientemente, los medios móviles (Hjorth \& Goggin, 2009; Goggin, 2010; Goggin \& Hjorth, 2014). Sin embargo, además de las oportunidades que ofrece Internet, los niños y niñas también experimentan riesgos evidenciando la interdependencia de ambos (Livingstone, Haddon, Görzig \& Ólafsson, 2011): cuanto más usan los menores internet, mayor es la gama de oportunidades que tienen y mayor es la probabilidad de exposición a experiencias de riesgo.

La experiencia digital de la infancia y la adolescencia se ha acentuado con el uso de smartphones y tabletas, así como con las smart $T V \mathrm{~s}$, videoconsolas, con los smartwatch, las aplicaciones que monitorizan la actividad fisica y otros recursos digitales que forman parte del Internet de las Cosas (Mascheroni \& Holloway, 2019). Estas tecnologías y plataformas les proporcionan a los menores recursos para el entretenimiento, la comunicación, el aprendizaje, la creatividad y la participación social, pero también plantean retos para los menores, sus progenitores, sus profesores, para la Administración y para todos aquellos profesionales que velan por su seguridad y bienestar. Las oportunidades (comunicación, aprendizaje, creatividad, entretenimiento, etc.) y los riesgos online (ciberacoso, discurso del odio, pornografía, grooming, explotación comercial, etc.) van de la mano: cuanto más usan internet los menores, más se benefician de sus oportunidades y adquieren más competencias, pero también se exponen más frecuentemente a riesgos (Livingstone et al., 2011).

En este sentido, los niños y niñas que están menos expuestos tanto a las oportunidades como a los riesgos tienden a sentirse más molestos y preocupados cuando sufren una experiencia online negativa (ibídem; ver también Livingstone, Hasebrink, \& Görzig, 2012). Tanto entre los mayores, expuestos a más riesgos pero más resilientes, como entre los pequeños, que participan en menos actividades y se enfrentan, por tanto, a menos riesgos, la vulnerabilidad online y offline van de la mano. La medición de las experiencias de riesgo y daño en línea está presente en la metodología y el marco operativo de EU Kids Online (Livingstone et al., 2011). Por eso el daño ha sido medido de forma subjetiva en función de lo que indicaban las respuestas de los niños y las niñas en las diferentes encuestas (2010, 2015 y 2018) ante las experiencias de riesgo. Podríamos sacar dos conclusiones. Una, se puede hablar de un ligero incremento en la probabilidad de que los menores afirmen que algo les ha molestado, lo que puede asociarse a la intensificación del uso a través de smartphones y tabletas para acceder a internet. Y dos, esta asociación refuerza la denominada «teoría del uso»: cuanto más usan internet los menores, disfrutan de más oportunidades, pero también se exponen a más experiencias de riesgo. 


\section{Cyberbullying, contactos con extraños, sexting, pornografía y otros riesgos}

Si bien es una cuestión recurrente tanto en la investigación como en las agendas públicas y políticas, no hay una definición estandarizada de lo que es el cyberbullying dado que el fenómeno en sí mismo está en permanente evolución (Schrock \& Boyd, 2008; ver también Levy et al., 2012). La mayoría se basan en la definición del propio bullying y sus componentes. El bullying ha sido descrito como una forma de agresión que es intencional, repetitiva, y que implica una desigualdad de poder entre víctima y agresor. Consecuentemente, el cyberbullying se define como una agresión intencional y repetida utilizando cualquier tipo de aparato tecnológico, como internet o el teléfono móvil. La investigación ha mostrado que las especificidades de la comunicación online o móvil refuerza los rasgos del bullying tradicional añadiéndole nuevos elementos. Por ejemplo, el anonimato «puede incrementar la naturaleza amenazadora de un acto de cyberbullying, o el sentido de impotencia de la víctima resultante» (Levy et al., 2012, p. 11), e igualmente incrementar el desequilibrio de poder entre la víctima y el agresor. El anonimato, sin embargo, puede no ser exclusivo de las comunicaciones en línea (y de hecho, el ambiente escolar puede facilitar que haya actos de bullying anónimos, como Levy y sus colegas señalan). Además, mientras un acto de cyberbullying puede no repetirse en el tiempo necesariamente (Levy et al., 2012), las propiedades de los públicos mediados — persistencia, trazabilidad, replicabilidad y audiencias invisibles (Boyd, 2008) - amplifican potencialmente la duración del cyberbullying y sus consecuencias dañinas, ya que audiencias más amplias pueden verse implicadas.

La investigación ha mostrado que, si bien el cyberbullying es menos común que el bullying offline (Livingstone et al., 2011; Ybarra \& Mitchel, 2014; Garmendia, Jiménez \& Larrañaga, 2019), es una experiencia muy angustiosa y dañina (Livingstone et al., 2011). El desplazamiento de los espacios offline a los online implica que las fronteras de espacio y tiempo van perdiendo su sentido: no se puede abandonar un espacio y suponer que el bullying terminará; de hecho, el bullying es más susceptible de ocurrir también tras la escuela, en diversas plataformas (Kernaghan \& Elwood, 2013). Además, comparadas con las formas de bullying cara a cara, las fronteras entre los roles de víctima, agresor y espectadores son más dificiles de trazar en el bullying online (Lampert \& Donoso, 2012). Con todo, y aun sin ánimo de subestimar la importancia cuantitativa de las cifras y del retrato que dibujan de una infancia y adolescencia enfrentada a problemas en la gestión de la convivencia, la duplicación de la población que dice haber sufrido acoso ha de entenderse en un contexto de preocupación social creciente respecto a las situaciones de abuso y desprotección de la infancia. Las iniciativas institucionales de cara a la sensibilización y el amparo de los menores afectados están relacionadas con una agenda mediática en la que términos como acoso escolar, bullying, cyberbullying o abusos entre menores son relativamente habituales. Hay una mayor atención pública al fenómeno, más campañas, más sen- 
sibilidad en definitiva. Y esto puede estar relacionado con mayores facilidades para identificar situaciones de abuso y acoso por parte de quienes las sufren, que quizá anteriormente sentían que el acoso escolar estaba normalizado o ni siquiera era considerado problemático («cosas de niños»).

Además, también es necesario tener en cuenta las dificultades inherentes a la definición del acoso y a lo que se considera, o no, como tal, y a la estrecha relación con la vivencia personal de cada una de las personas que se consideran víctimas de un abuso: bajo la etiqueta de bullying caben desde situaciones vejatorias objetiva e indiscutiblemente graves, también hay lugar para otras situaciones que dependiendo de la sensibilidad y la resiliencia de cada víctima revisten una gravedad relativa. Con respecto al papel que juegan las tecnologías de la comunicación en este incremento en el número de personas que se autodefinen como víctimas del acoso, cabe señalar que el incremento de los casos de acoso cara a cara es notablemente mayor que el incremento de los casos de cyberbullying concretados a través de internet.

Los menores también están utilizando internet y los teléfonos móviles como parte de sus interacciones y exploraciones sexuales (Lenhart, 2009; Livingstone et al., 2011). Esta práctica ha sido denominada «sexting» (a partir de las palabras sex y texting), y ha sido definida de diversas maneras. Una aproximación restringe el sexting al intercambio de imágenes por medio del teléfono móvil. Lenhart define sexting como «crear, compartir y difundir imágenes de desnudos o semidesnudos sexualmente sugerente» de sí mismo o de alguien a quien se conoce, a través del móvil (2009, p. 2), excluyendo por tanto textos sexualmente insinuantes así como el resto de las plataformas de comunicación. La encuesta EU Kids Online, por el contrario, adoptó una noción del sexting más inclusiva, que tiene en cuenta las imágenes y los textos, y considera todo tipo de comunicación online más allá del uso de los teléfonos móviles (Livingstone et al., 2011).

El estudio Pew Internet Study (Lenhart, 2009) identifica tres supuestos básicos de sexting, cuando el intercambio de imágenes sexuales ocurre: (a) como parte de la experimentación adolescente con la identidad sexual y la intimidad, aunque sus protagonistas no sean todavía sexualmente activos; (b) entre dos compañeros, como parte de una relación sexual; c) como el prólogo de una actividad sexual, entre dos personas que aún no tienen una relación, de las cuales al menos una sí querría tenerla. De hecho, la mayoría del sexting puede ser contextualizado dentro de una relación de pares, como forma de «moneda de cambio» en la relación (Lenhart, 2009, p. 8). Sin embargo, las especificidades tecnológicas y la disponibilidad social de las TIC puede amplificar las fronteras, significados y audiencias del sexting: imágenes y textos intercambiados en el contexto de relaciones románticas, mediante mensajería instantánea (WhatsApp, Snapchat, etc.) o redes sociales pueden ser fácilmente distribuidas, publicadas en más espacios online y por tanto ser compartidas con audiencias más amplias. Por tanto, los mensajes sexuales pueden tener consecuencias no inten- 
cionadas y pueden convertirse en experiencias desagradables o problemáticas para algunos menores. La investigación precedente sostiene que el intercambio de imágenes, mensajes o invitaciones sexualmente explícitos está ligado al hostigamiento y el bullying, y por tanto conduce a una forma de "ciberbullying sexual» (Kofoed \& Ringrose, 2012; Ringrose, Gill, Livingstone, \& Harvey, 2012).

Por otra parte, una de las mayores fuentes de ansiedad en relación con las comunicaciones online de los jóvenes se refiere a lo que en inglés se define como stranger danger, esto es, la idea de que la gente joven puede conocer a alguien a través de internet, ser persuadida para contactar offline y terminar siendo víctima de abusos en ese encuentro cara a cara. De hecho, investigaciones precedentes han sugerido que «conocer extraños» puede abarcar diversas circunstancias y experiencias, que no pueden ser asumidas como universalmente problemáticas (Barbovschi et al., 2012; Ito et al., 2009); al mismo tiempo, estudios previos muestran que el riesgo de ser dañado en un encuentro cara a cara con alguien que se ha conocido online es bajo ( $\mathrm{Li}$ vingstone et al., 2011; Garmendia, Jiménez, Karrera, Larrañaga, Casado, Martínez, \& Garitaoanadia, 2019).

Una de las principales razones radica en el modo en el que funciona la sociabilidad en línea, a través de la cual los menores tienden a extender su red de contactos activando «vínculos latentes» (por ejemplo, personas con las que comparten amigos, hobbies o ubicación), más que buscando a personas con las que no guardan conexión alguna en el entorno offline. De hecho, la mayoría de los encuentros cara a cara con personas previamente contactadas online son con «amigos de amigos» y no con completos desconocidos (Barbovschi et al., 2012). Por tanto, el primer paso es entender cómo funciona la comunicación en línea y el proceso de contacto con personas que se han conocido por internet, y seguidamente identificar cómo se pasa a encontrarse offline con alguien que se conoció online.

El contacto con personas que se conocen a través de internet, per se, no tiene por qué ser negativo o implicar riesgos. De hecho, en ocasiones proporciona a los menores la oportunidad de compartir intereses y aficiones (Ito et al., 2009). Por otra parte, no todos los contactos online forzosamente conducen a encuentros offline, y lo que es más importante, no todo encuentro cara a cara con alguien que se conoció en internet tiene consecuencias nocivas. Considerando que la relación entre riesgo y daño es compleja y no lineal, incluso en situaciones en las que encontrarse fisicamente con contactos online no resulte dañina, siempre resultan oportunas las campañas que buscan concienciar al respecto, así como programas de seguridad que promueven una gestión responsable de los contactos a través de internet.

La pornografia, y más concretamente, la influencia negativa que ejerce la pornografia en los menores, es un objeto de estudio controvertido. La creencia de que la ausencia de censura y, en consecuencia, las facilidades para que el contenido por- 
nográfico circule por internet haya llevado la pornografia de «debajo de la cama» a la pantalla genera ansiedades públicas (Rovolis \& Tsaliki, 2012, p. 173). Sin embargo, la ubicuidad de los contenidos sexuales en internet se ha abordado en múltiples estudios (ver, entre otros, Ey \& Cupit, 2011) y, por ejemplo, el proyecto EU Kids Online reveló que uno de cada cuatro menores se ha encontrado con contenido pornográfico, y sólo el $14 \%$ se han encontrado, accidental o intencionalmente, con imágenes sexuales online (Livingstone et al., 2011). Igualmente, los datos también mostraron que mientras ver imágenes sexuales es más común entre chicos y adolescentes de mayor edad, los niños más pequeños y las chicas tienden en mayor medida a disgustarse por los contenidos con los que se encuentran.

En general, solo uno de cada tres menores expuestos a contenido sexual online manifestó sentirse molesto tras esta experiencia, aunque existe una considerable variación intercultural (ibídem). A partir de estos hallazgos Rovolis y Tsaliki (2012) concluyen que, tal y como las aproximaciones desde los estudios culturales han estado defendiendo durante un tiempo (Attwood \& Smith, 2011; Buckingham \& Bragg, 2004), la preocupación por los efectos negativos de la pornografia se sobredimensiona en los medios de comunicación.

Es innegable que internet, y en particular el internet móvil y los dispositivos de uso personal, más complicados de supervisar por parte de las familias, han facilitado el acceso a contenidos que en muchas ocasiones pueden considerarse incluso pornográficos. A este respecto, cabe subrayar el modo en el que el acceso a imágenes sexuales por medio del teléfono móvil se ha multiplicado por diez en los últimos cinco años, y el acceso a través de páginas web se ha duplicado ampliamente. Como se ha mencionado antes, esto está relacionado con el aumento de la dotación de teléfonos móviles y del acceso, en conjunto, a todos los recursos de internet.

Además, internet ha permitido la circulación sin precedentes de una ingente cantidad de contenidos generados por los propios usuarios y usuarias, que no son producidos ni distribuidos masivamente por empresas, sino que son realizados por personas particulares (UGC, User-Generated Content). Si bien es cierto que la creación y la posibilidad de compartir contenidos es una de las oportunidades fundamentales que ofrece la red, hay ciertos contenidos generados por usuarios que podrían considerarse problemáticos: los que promueven desórdenes alimenticios; comportamientos auto-lesivos, o consumo de drogas, o los materiales que promueven la discriminación y la violencia contra ciertos grupos están entre los principales ejemplos de contenidos negativos generados por usuarios. Aunque hay ciertas evidencias de que la exposición a este tipo de mensajes es relativamente común entre los menores (Livingstone et al., 2011), esta cuestión ha recibido menos atención por parte de legisladores e investigadores que otras como el bullying, el sexting, el contacto con extraños o la pornografía. 
Esto incluye: riesgos comerciales, como perder dinero como consecuencia de ser víctimas de fraudes online; riesgos técnicos, como virus o software malintencionado; y riesgos relacionados con el mal uso de la información personal. Esto último comprende ser víctima del hackeo de la cuenta de correo electrónico o el perfil en redes sociales; el uso inadecuado de información personal y de fotos de la víctima que un tercero se atribuye (por ejemplo, mediante la creación de perfiles falsos); y personas que se hacen pasar por otras, o catfishing. Mientras la literatura sobre esta cuestión es aún dispersa, hay algunas evidencias de que el abuso y el mal uso de la información personal merecen atención. De acuerdo con los datos de EU Kids Online, el 9\% de los menores de 11 a 16 años han experimentado una o más de las tres formas de mal uso de la información personal que se investigaban, siendo las experiencias más habituales que alguien utilizara la contraseña de los niños o intentara hacerse pasar por ellos.

Según nuestra encuesta de 2018, los virus constituyen el riesgo con el que se encuentran más menores, uno de cada cuatro, que además se incrementa con la edad, pasando del 13\% entre los niños más pequeños al 32\% de los mayores. Por el contrario, que el teléfono se haya infectado por un virus ha sido reportado por una minoría de menores (5\%), siendo más habitual entre los más mayores, lógicamente.

Entre los riesgos asociados al mal uso de la información personal, es equivalente el porcentaje de menores que han experimentados riesgos relacionados con la privacidad habiendo detectado que alguien utilizó su información personal de una forma que no les gustó, o habiendo detectado que alguien usó su password o su teléfono para acceder a su información o suplantó su identidad. Si bien los datos señalados pueden considerarse satisfactorios por lo limitados, no deberíamos minimizar la relevancia de los riesgos asociados al mal uso de los datos personales; tal y como muestran las evidencias cualitativas en las que también hemos trabajado, los menores son particularmente sensibles a las cuestiones relacionadas con la privacidad (Garmendia et al., 2016).

\section{Reaccionando a los riesgos}

La mayoría de las experiencias online no tienen por qué ser dañinas, y de hecho los menores no tienen por qué percibirlas como peligrosas o problemáticas (Livingstone et al., 2012; Vandoninck, d'Haenens \& Roe, 2013). Sin embargo, cuando se encuentran con experiencias negativas en internet, los menores despliegan una serie de estrategias para adaptarse a la situación y reducir el estrés emocional y psicológico derivado de la misma. Afrontar riesgos online implica, para los niños y las niñas, adoptar «estrategias de resolución de problemas específicos de internet» tras una experiencia en línea negativa (Vandoninck et al., 2013, p. 61). La encuesta EU Kids Online (Livingstone et al., 2011) identificaba tres tipos de estra- 
tegias para afrontar los riesgos: respuestas pasivas, que incluyen actitudes resignadas (dejar de utilizar internet durante un tiempo) y auto-acusatorias (sentirse culpable por lo que ha ocurrido); respuestas proactivas (como reportar o denunciar contenidos inadecuados o contactos inadecuados, bloquear a los contactos no deseados, etc.); y respuestas comunicativas (hablar sobre lo sucedido con los padres y madres, con pares, profesores u otros adultos de confianza). Aprender cómo gestionar las experiencias negativas de una manera eficaz, así como detectar cuáles son las respuestas más adecuadas y efectivas para cada ocasión, forma parte del proceso de construcción de la resiliencia (Vandoninck et al., 2013; Vandoninck \& d'Haenens, 2018) y de adaptación al entorno.

La estrategia para afrontar los riesgos online más habitualmente adaptada por los menores pasa por buscar apoyo en su entorno social, aunque en la mayoría de los casos tienden a combinar dos estrategias (Livingstone et al., 2011). La investigación previa ha probado que los menores que reciben un apoyo mayor por parte de sus pares son más resistentes a las experiencias negativas en internet, y que tanto los padres como el profesorado están en la posición de mediar en el desarrollo de la resiliencia si activamente se implican en las actividades online de los menores y les hacen recomendaciones relativas a la seguridad en internet (Vandoninck et al., 2013).

Para las niñas y los niños más pequeños es más probable hablar con su padre o su madre que con cualquier otro interlocutor, destacando que tanto para niños como para niñas la probabilidad de hablar con la madre es mayor. La importancia de padres y madres como fuente primaria de apoyo en caso de experiencias desagradables disminuye con la edad, y particularmente entre los niños. Paralelamente, los adolescentes son más propensos que los niños más pequeños a recurrir a sus pares para buscar apoyo. El género marca variaciones de importancia: las chicas adolescentes tienden a hablar con sus amigos, y más aún con sus madres y, en general, las chicas consideran con más probabilidad hablar con todos los interlocutores sugeridos salvo con el padre. Si bien en general los menores no consideran hablar con sus profesores, los chicos y chicas pre-adolescentes se inclinan a señalarlos como fuente de apoyo en mayor medida que los adolescentes, que apenas los consideran como posibles interlocutores. La probabilidad de hablar con personas dedicadas a ayudar a los menores, hermanos y hermanas u otros familiares o adultos en los que se confia también disminuye, aunque a ritmos diferentes, según aumenta su edad. Los menores valoran particularmente el papel de padres, madres y el de sus iguales, con lo cual estos agentes deberían ser muy considerados en las iniciativas de regulación. Por otra parte, aún hay un 23\% de menores (Garmendia et al., 2019) que afirma que no habló con nadie después de una experiencia desagradable en internet. Al diseñar políticas de acción al respecto las autoridades deberían de asegurarse que todos los niños y las niñas encuentren el apoyo social necesario, del tipo que sea. 


\section{Redes sociales}

El potencial de las redes sociales para facilitar el contacto con los iguales ha reforzado la preocupación pública respecto a los problemas derivados de internet, así como sobre el frágil equilibrio entre privacidad e intimidad en el contacto con las personas que se llegan a conocer a través de la red. Además, los smartphones y los dispositivos móviles en general amplían la gama de actividades comunicativas y el tipo de audiencias con las que los menores pueden entrar en contacto (Bertel \& Stald, 2013). La reconfiguración de estas actividades plantea nuevas cuestiones tales como: ¿puede aún considerarse el teléfono móvil como la herramienta de acceso permanente a la vida íntima? — «the full-time intimate sphere»- (Ling, 2008; Matsuda, 2005).

Aunque Instagram y Facebook son las redes sociales más utilizadas, el acceso a redes se diversifica con el uso de otras opciones. Los niños y las niñas simultanean el uso de varias plataformas para diversas actividades con el fin de contactar con diferentes audiencias. Además, diferentes redes sociales pueden implicar distintas nociones de amistad y diversos regímenes de privacidad y apertura. Adoptando la noción de «repertorio comunicativo» (Haddon, 2004), asumimos que los menores, igual que los adultos, desarrollan sofisticados repertorios relacionados con los hábitos, dispositivos y servicios de entre los cuáles eligen en cada caso el que mejor encaja en la situación y relación comunicativa. Más que sustituir una red social por otra, las combinan e integran en sus prácticas comunicativas.

Las redes sociales también les dan a los menores una libertad que no tienen en su vida diaria, ya que las redes les proporcionan un espacio de sociabilidad alejado del control familiar y escolar, entornos donde ellos y ellas pasan la mayor parte de su tiempo. Además, aunque en la última década ha descendido de una forma apreciable el porcentaje de personas que les resulta más fácil ser ellos mismos online que cara a cara (ver Livingstone et al., 2013), todavía en nuestra encuesta de 2018 tiene una frecuencia del $22 \%$.

Los adolescentes y los adultos jóvenes son especialmente vulnerables, en particular, a los efectos de las redes sociales y, en general, a la comunicación digital: son a la vez los primeros en adoptar las TICs, son usuarios ubicuos y altamente susceptibles a las influencias de sus compañeros (Lenhart, 2015). En todo caso, desde las primeras investigaciones (Nie, 2001) se plantearon las primeras preocupaciones que el uso de las redes sociales podría traer: el aumento de los niveles de aislamiento de las personas y el crecimiento de relaciones superficiales que tendría un impacto en la habilidad personal para la socialización en el mundo real. Sin duda, también ha tenido aspectos muy positivos. Las redes sociales se han ido convirtiendo poco a poco, en los últimos años en populares avenidas para la comunicación sobre salud y su promoción. Han supuesto también una oportunidad para para llegar a la juventud y ayudar a los ado- 
lescentes y progenitores a evitar la progresión de comportamientos adictivos (alcohol, tabaco, marihuana y juego) (Romer et al., 2017). En el caso del maltrato infantil, por ejemplo, que es uno de los problemas más significativos de salud en USA (3,6 millones de casos referidos al año), las redes sociales y en particular Facebook, está siendo usada para la promoción de los programas de prevención del maltrato infantil (behavioral parent training programs, BPTs) y la mayoría de los progenitores de la investigación desarrollada por Edwards-Gaura et al. (2014) valoran positivamente Facebook en particular, usan la red social y tienen una cuenta. En ese ámbito social, los jóvenes usan las redes sociales para negociar su identidad sexual y de género de formas complejas y diversas, desde bromas y coqueteo, hasta la intimidad o la agresión. Y unos grupos se benefician más que otros. Adolescentes que se identificaron como gays, lesbianas, bisexuales u otros pertenecientes al colectivo LGTB usaron las redes sociales (social networking sites) para el desarrollo de su identidad y la comunicación más que sus pares heterosexuales (Lim et al., 2019). Pero también las redes sociales tienen efectos negativos. Es el caso de la publicitación de las bebidas energéticas en las plataformas digitales, Facebook, Twitter y Youtube, como evidencian el amplio número de usuarios comprometidos con likes, shares, posts, tweets, videos, advergames y comentarios, ha sido uno de los factores clave para incentivar el sobrepeso y la obesidad de los jóvenes (Buchanan et al., 2018). La comercialización de bebidas alcohólicas es también común en las redes sociales. Muchas marcas están presentes en los social networks, lo que les brinda la oportunidad de conectarse con los jóvenes y desarrollar la fidelidad a la marca. Aunque el acceso requiere verificación de la edad antes de permitir a los usuarios acceder al contenido online, esas restricciones son dificiles de hacer cumplir y se sortean con facilidad. De hecho, algunos países, entre ellos el Reino Unido, han aprobado medidas regulatorias restringiendo el marketing de bebidas y comidas no saludables (The Food Foundation, 2017).

Por otra parte, está relativamente extendida la difusión en redes sociales de videos, imágenes e informaciones de menores por sus progenitores, profesores y amigos, sin su aprobación o consentimiento (sharenting).

Existe una creciente concienciación sobre los riesgos online entre padres y madres y menores que se manifiesta en una mayor participación de los progenitores en la mediación de la seguridad de los menores online, y en el desarrollo de habilidades de seguridad o la adopción de medidas preventivas entre los menores. El acoso continúa siendo el riesgo que más daño causa a quienes lo sufren, pero pese a un cierto discurso social y mediático y a las posibilidades que las redes sociales dan para amplificar situaciones de abuso, aún son muchos más los casos de cyberbullying que son una prolongación del cara a cara que los que, en palabras de los afectados, tienen su origen en el espacio online.

La mediación de la familia es fundamental para prevenir y gestionar los riesgos en internet y los usos inadecuados. En casi todos los grupos observados se detec- 
tan carencias en esa área, y en muchas ocasiones están relacionados con la baja alfabetización digital de sus progenitores. Las herramientas digitales para la socialización pueden ser de utilidad para facilitar su integración: redes como WhatsApp (y otras) les permite socializar con sus compañeros de clase al margen de la escuela.

En cualquier caso, y a pesar de que los menores son más conscientes de los peligros asociados al ciberacoso $\mathrm{u}$ otras situaciones potencialmente conflictivas, sigue siendo necesario promover usos de la comunicación móvil más seguros y responsables. Esto podría pasar por incrementar la conciencia sobre las cuestiones relacionadas con la privacidad, el uso de las aplicaciones diseñadas para denunciar o bloquear, las funciones vinculadas al control y la geolocalización o los riesgos asociados a la escalada de intercambios que a veces se dan en casos de conflictos online (Marwick \& Boyd, 2014).

\section{Referencias bibliográficas}

Attwood, F. \& Smith, C. (2011). Investigating young people's sexual cultures: an introduction. Sex Education, 11(3), pp. 235-242. DOI: 10.1080/14681811.2011.590040

Barbouschi, M. Marinescu, V. Velicu, Anca \& Laszlo, E. (2012). Meeting new contacts online. In Livingstone, S. Haddon, L. \& Görzig, A. (Ed.), Children, risk and safety on the internet: Research and policy challenges in comparative perspective. (pp. 177-189). Bristol: Policy Press.

Bertel, T. \& Stald, G. (2013). From SMS to SNS: the use of the internet on the mobile phone among young Danes. En K. Cumiskey y L. Hjorth (eds.), Mobile media practices, presence and politics. The challenge of being seamlessly mobile, 198-213. New York: Routledge.

Boyd, D. (2008). Why youth (heart) social network sites: the role of networked publics in teenage social life. In D. Buckingham (ed.), Youth, identity and digital media (pp. 119142). Cambridge, MA: MIT Press.

Buchanan, L., Yeatman, H., Kelly, B., \& Kariippanon, K.A Thematic content analysis of how marketers promote energy drinks on digital platforms to young Australians. Australian and New Zealand Journal of Public Health. 2018; 42:530-2; doi: 10.1111/1753-6405.12840

Buckinggham, D. \& Bragg, S. (2004). Young people, sex and the media, Basingstoke. Palgrave Macmillan.

Edwards-Gaura, A., Whitaker, D., \& Self-Brown, S. (2014). Can Social Networking Be Used to Promote Engagement in Child Maltreatment Prevention Programs? Two Pilot Studies. Western Journal of Emergency Medicine, Volume XV, no. 5, pages 575-581. Available through open access at. DOI: 10.5811/westjem.2014.4.21413.

Ey, L-A. \& Cupit, C.G. (2011). Exploring young children's understanding of risk associated with Internet usage and their concepts of management strategies. Journal of Early Chilhood Research 23. https://doi.org/10.1177/1476718X10367471. 
Garmendia, M. Jiménez, E., Casado, M.A. \& Mascheroni, G. (2016). Net Children Go Mobile: Riesgos y oportunidades en internet y el uso de dispositivos móviles entre menores españoles (2010-2015). Madrid: Red.es/Universidad del País Vasco/Euskal Herriko Unibertsitatea.

Garmendia, M., Jiménez, E., Karrera, I., Larrañaga, N., Casado, M.A., Martínez, G. \& Garitaoanadia, C. (2019). Actividades, Mediación, Oportunidades y Riesgos online de los menores en la era de la convergencia mediática. León: Instituto Nacional de Ciberseguridad (INCIBE)

Goggin, G. (2010). Global mobile media. New York: Routledge.

Goggin, G. \& Hjorth, L. (2014). The Routledge Companion to Mobile Media. New York: Routledge.

Haddon, L. (2004). Information and communication technologies in everyday life. Oxford: Berg.

Hjorth, L. \& Goggin, G. (2009). Mobile technologies: From telecommunications to media. London: Routledge.

Ito, M. et al. (2009). Hanging out, messing around and geeking out: Kids living and learning with new media. Cambridge, MA: MIT Press.

Kernaghan, D., \& Elwood, J. (2013). All the (cyber) world's a stage: Framing cyberbullying as a performance. Cyberpsychology: Journal of Psychosocial Research on Cyberspace, 7(1), article 5. http://dx.doi.org/10.5817/CP2013-1-5

Kofoed, J. \& Ringrose, J. (2012). Travelling and sticky affects: Exploring teens and sexualized cyberbullying through a Butlerian-Deleuzian-Guattarian lens. Discourse: Studies in the Cultural Politics of Education, 33(1), 5-20.

Lampert, C. \& Donoso, V. (2012). Bullying. In Livingstone, S. Haddon, L. \& Görzig, A. (ed.), Children, risk and safety on the internet: Research and policy challenges in comparative perspective. (pp. 165-176). Bristol: Policy Press.

Lenhart A. (2015). Teens, Social Media \& Technology Overview. Available at: https:// www.pewresearch.org/wp-content/uploads/sites/9/2015/04/PI_TeensandTech_ Update2015_0409151.pdf.

Lenhart, A. (2009). Teens and sexting: How and why minor teens are sending sexually suggestive nude or nearly nude images via text messaging. Washington, DC: Pew Research Center. Retrieved from: http://pewresearch.org/assets/pdf/teens-and-sexting.pdf.

Levy, N., Cortesi, S., Crowley, E., Beaton, M., Casey, J., \& Nolan, C. (2012). Bullying in a networked era: A literature review. Berkman Center Research Publication, 17. Retrieved from http://cyber.law.harvard.edu/publications/2012/kbw_bulling_in_a_networked_era.

Lim MSC, Cooper S., Lewis L. et al. (2019). Prospective mixed methods study of online and offline social networks and the development of sexual agency in adolescence: the Social Networks and Agency Project (SNAP) protocol. BMJ Open 2019; 9: e024329. doi:10.1136/ bmjopen-2018-024329

Ling, R.S. (2008). New tech, new ties. Cambridge, MA: MIT Press.

Livingstone S., Kirwil L., Ponte C. et al. (2013). In their own words: what bothers children online? with the EU Kids Online Network. London, UK: London School of Economics and Political Science. 
Livingstone, S., Haddon, L., Görzig, A., \& Ólafsson, K. (2011). Risks and safety on the internet: The perspective of European children. Full findings. London: LSE, EU Kids Online.

Livingstone, S., Hasebrink, U. \& Görzig, A. (2012). Towards a general model of determinants of risks and safety. In S. Livingstone, L. Haddon, \& A. Görzig (eds.) Children, risk and safety on the internet (pp. 323-339). Bristol: Policy Press.

Mascheroni, G. \& Holloway, D. (2019). The quantified child: Discourses and practices of dataveillance in different life stages. En Erstad, O., Flewitt, R., Kümmerling-Meibauer, B. and Pires Pereira, I. (Eds.) The Routledge Handbook of Digital Literacies in Early Childhood. London: Routledge

Mascheroni, G. \& Ólafsson, K. (2014). Net Children Go Mobile: Risks and opportunities (2nd edn). Milano: Educatt.

Mascheroni, G., \& Holloway, D. (eds.) (2017). The Internet of Toys: A report on media and social discourses around young children and Io Toys. DigiLitEY.

Mascheroni, G., \& Ólafsson, K. (2018). Accesso, usi, rischi e opportunità di internet per i ragazzi italiani. I primi risultati di EU Kids Online 2017. EU Kids Online e OssCom.

Marwick, A., \& Boyd, D. (2014). «It's just drama»: teen perspectives on conflict and aggression in a networked era. Journal of Youth Studies. DOI: 10.1080/13676261.2014.901493

Matsuda, M. (2005). Mobile communication and selective sociality. En M. Ito, D. Okabe, y M. Matsuda (Eds.) Personal, portable, pedestrian. Mobile phones in Japanese life, 23-142. Cambridge, MA: MIT Press.

Nie, N.H. (2001). Sociability, interpersonal relations and the internet: reconciling conflicting findings. American Behavioural Scientist, 45 (3), 420-435.

Ringrose, J., Gill, R., Livingstone, S., \& Harvey, L. (2012). A qualitative study of children, young people and 'sexting': A report prepared for the NSPCC. London: National Society for the Prevention of Cruelty to Children.

Romer, D., \& Moreno, M. (2017). Digital Media and Risks for Adolescent Substance Abuse and Problematic Gambling. PEDIATRICS Volume 140, number s2. DOI: https:// doi. org/ 10. 1542/ peds. 2016-1758L

Rovolis, A. \& Tsaliki, L. (2012). Harm, childhood and appropriate media content: an ongoing debate. In Livingstone, S. Haddon, L. \& Görzig, A. (Ed.), Children, risk and safety on the internet: Research and policy challenges in comparative perspective. (pp. 165-176). Retrieved from https://bit.ly/2YYGKct

Schrock, A. \& Boyd, D. (2008). Online threats to youth: Solicitation, harassment, and problematic content: Literature review prepared for the Internet Safety Technical Task Force. Retrieved from http://cyber.law.harvard.edu/sites/cyber.law.harvard.edu/files/RAB_Lit_ Review_121808_0.pdf

The Food Foundation (2017). UK'S RESTRICTIONS ON JUNK FOOD ADVERTISING TO CHILDREN. Disponible en: www.foodfoundation.org.uk. 
Vandoninck, S. \& d'Haenens, L. (2018). Resiliencia online: la capacidad de minimizar el impacto de los riesgos y de gestionar las situaciones potencialmente dañinas en internet. En Jiménez, E., Garmendia, M. y Casado, M.A. (eds.) Entre selfies y whatsapps. Oprtunidades y riesgos para la infancia y la adolescencia conectada, 189-208. Barcelona: Gedisa.

Vandoninck, S., d'Haenens, L., \& Roe, K. (2013). Online risks: Coping strategies of less resilient children and teenagers across Europe. Journal of Children and Media, 7(1), 60-78.

Ybarra, M.L. \& Mitchel, J.M. (2014). «Sexting» and Its Relation to Sexual Activity and Sexual Risk Behavior in a National Survey of Adolescents. Journal of Adolescent Health, 55, 757-764. 